\title{
Optimal Xeno-free Culture Condition for Clinical Grade Stem Cells from Human Exfoliated Deciduous Teeth
}

\author{
Nathaphon Tangjit ${ }^{1}$, Surachai Dechkunakorn ${ }^{1}$, Niwat Anuwongnukroh ${ }^{1}$, \\ Anuson Khaneungthong ${ }^{1}$, Hathaitip Sritanaudomchai ${ }^{2}$ \\ Departments of ${ }^{1}$ Orthodontics, ${ }^{2}$ Oral Biology, Faculty of Dentistry, Mahidol University, Bangkok, Thailand
}

Background and Objectives: Stem cells from human exfoliated deciduous teeth (SHED) are a promising clinical resource for various tissue defects, including lumbar spondylosis, neural compression, and cleft palate. Use of media containing animal-derived serum carries potential risk of infectious diseases and unwanted immunogenicity. To increase the potential utility of SHED for clinical application, SHED was adapted to xeno-free conditions.

Methods: Define xeno-free culture media were compared with the conventional serum containing media in the culture of SHED. Cultured SHED in different media were further characterized through proliferative capacities, cellular phenotype, and differentiation potential.

Results: Selected xeno-free media were capable of supporting the growth of SHED. MSCGM-CD Bulletkit medium greatly increased the number and proliferate capacity of colony-forming unit-fibroblast than SHED cultured in other media. In addition, the characteristic surface markers expression and multipotent differentiation potential of SHED in the MSCGM-CD Bulletkit medium were comparable to those observed with serum-containing medium.

Conclusions: The xeno-free medium described herein has the potential to be further used for the safe expansion and to determine efficient way to produce clinical grade dental stem cells for therapeutic applications.

Keywords: Xeno-free media, Dental pulp stem cells, Clinical grade cells, Proliferation, Primary tooth, Osteogenesis

\section{Introduction}

The discovery of the mesenchymal stem cells (MSCs) present in the pulp of human exfoliated deciduous teeth (SHED) (1) has been an exciting finding in the field of regenerative medicine, which has a significant value in im-

Received: August 12, 2017, Revised: November 14, 2017, Accepted: January 25, 2018, Published online: February 28, 2018 Correspondence to Hathaitip Sritanaudomchai

Department of Oral Biology, Faculty of Dentistry, Mahidol University, 6 Yothi Road, Rajthawee, Bangkok 10400, Thailand Tel: +66-2-200-7849, Fax: +66-2-200-7848

E-mail: hathaitip.sri@mahidol.ac.th

(c) This is an open-access article distributed under the terms of the Creative Commons Attribution Non-Commercial License (http://creativecommons.org/ licenses/by-nc/4.0/), which permits unrestricted non-commercial use, distribution, and reproduction in any medium, provided the original work is properly cited.

Copyright (C) 2018 by the Korean Society for Stem Cell Research proving the life of patients suffering from various diseases. SHED is self-renewable and also has the ability to develop into many types of body tissues as compared to other stem cells. They can provide alternative treatment options in regenerative medicine since they can be used for not only to dental tissue regeneration, but also to facilitate repair of non-dental tissues, such as bone and nerves $(2,3)$. SHED have higher proliferation rates, and form sphere-like clusters and differentiate into osteoblasts. These cells have been reported to repair calvarial defects in mice due to their ability to differentiate into osteoblasts. SHED secretes neurotrophic factor for the repair of motor neurons following dental injury and, therefore, it was proposed that SHED could be useful in the treatment of neurodegenerative diseases (4). Moreover, SHED is ethically noncontroversial, noninvasive, less dependent on timing, and far less expensive.

Salutary application of MSCs requires for a wide range 
of human conditions demands in order to reach appropriate cell numbers that can achieve therapeutic outcomes. Thus, identification of optimal culture conditions is a prerequisite for MSC clinical applications $(5,6)$. Addition of fetal bovine serum (FBS) to culture media provides the cells with vital nutrients, attachment factors, and growth factors. However, utilization of animal derived products bears critical limitations and safety concerns (7), such as animal derived (xeno) antigens and infectious agents present in FBS that can lead to a risk of infusion reactions and transmission of zoonotic diseases to the recipient of MSCs therapy (8).

To avoid undesirable complications, alternative animal product-free media formulations have been evaluated. In this study, we compared the phenotype, growth potential, and characteristics of MSCs derived from the pulp of human exfoliated deciduous teeth on available mediaMSCGM-CD Bulletkit, MSC Growth Medium DXF, and Xuri MSC Medium Serum-free/Xeno-free with media supplemented with FBS.

\section{Meterials and Methods}

\section{Ethics statement}

The study was approved by the Ethical Committee on Human Rights Relate to Human Experimentation of Faculty of Dentistry, Mahidol University (IRB 2014/ 041.2110). Human exfoliated deciduous teeth were collected from children ( $6 \sim 8$ years of age) at the Faculty of Dentistry, Mahidol University. Teeth with previous restorations, history of trauma, and/or signs of pulpal pathology, were excluded from this study.

\section{Isolation and cell culture of SHED}

Fragments of dental pulp tissues were extracted and submerged in culture medium using $25 \mathrm{~mm}^{2}$ culture flasks. The culture media consisted of Dulbecco's modified Eagle's medium (DMEM) with high glucose (Hyclone, Logan, UT, USA), 10\% fetal bovine serum (FBS; Biochrom $\mathrm{GmbH}$, Berlin, Germany), and 1\% penicillin/streptomycin (Gibco, Rockville, MD, USA). The dental pulp stem cells were obtained using the outgrowth methods and incubating condition of $37^{\circ} \mathrm{C}$ in a humidified atmosphere containing $5 \% \mathrm{CO}_{2}$. The medium was changed every 2 days and the cell cultures were monitored regularly with an inverted phase-contrast microscope. Upon reaching 80\% confluence, cells were harvested using 0.05\% Trypsin/ EDTA (Gibco) and sub-cultured for further experiments. For each assay, the stem cells of three different donors were used.

\section{Human SHED expansion}

The culture media with FBS supplement or xeno-free media, MSC Growth Medium DXF (DXF; PromoCell, Heidelberg, Germany), MSCGM-CD Bulletkit (MSCGM-CD, Lonza, Allendale, NJ, USA), and Xuri MSC Medium Serum-free/Xeno-free (Xuri, GE Healthcare Life Sciences, Glattbrugg, Switzerland), were used to support the expansion of human SHED. After cells harvesting with Trypsin/EDTA, the cell pellets were resuspended in a 30:70 ratio of xeno-free medium:culture medium with FBS and maintained on human plasma fibronectin $\left(5 \mu \mathrm{g} / \mathrm{cm}^{2}\right.$; Merck Millipore, Ontario, Canada)-coated T-75 cell culture flask. Subsequently, cells were gradually allowed to adapt to xeno-free media, using an increasing proportion of xeno-free medium (with ratios of xeno-free medium to culture medium with FBS at 50:50 and 70:30) up to 100\% during the first week of culture. Throughout the duration of the cultures, individual culture flasks remained separate and were propagated individually.

\section{Cell proliferation}

The SHED was seeded into 96-well plates at 2,000 cells/well in culture medium with FBS or xeno-free media (DXF, MSCGM-CD and Xuri). After 1, 4, 6, 8, and 12 days in culture, cells were stained with sulforhodamine B (SRB; Sigma St. Louis, MO, USA). The amount of dye extracted from stained cells was directly proportional to the number of cells for the measurement of cell proliferation.

\section{Colony-Forming Unit-Fibroblast (CFU-F) assay}

The SHED expanded in culture to $80 \sim 90 \%$ confluency were harvested with Trypsin/EDTA and counted using a haemocytometer. Harvested cells were reseeded in triplicates into a 6-well plate at 500 cells/well (in culture medium with FBS or xeno-free media). After 14 days, cultures were washed with PBS (Biochrom $\mathrm{GmbH}$, Berlin, Germany) and stained with $0.1 \%$ toluidine blue $\mathrm{O}$ (Scharlab S.L., Barcelona, Spain) in paraformaldehyde (Sigma) for 15 minutes at room temperature (RT). The cells were washed with distilled water and the colony morphology was inspected under a light microscope. Colonies larger than $2 \mathrm{~mm}$ in diameter were enumerated using ImageProPlus 7.0 program (Media Cybernetics, Rockville, MD, USA).

\section{Immunophenotypic characterization of SHED}

For the cell surface antigen analysis, SHED were harvested, washed in cold PBS supplemented with 0.5\% Bovine Serum Albumin (BSA, Sigma), and aliquots of $5 \times 10^{5}$ cells were labeled (for $30 \mathrm{~min}$ in the dark at $4^{\circ} \mathrm{C}$ ) 
with monoclonal antibodies specific for human markers associated with mesenchymal and hematopoietic lineages. Namely, mouse anti-human antibodies against the following antigens were used: CD34 (FITC conjugated), CD44 (FITC conjugated), CD73 (APC conjugated), and CD105 (PE conjugated) (all purchased from BioLegend, San Diego, CA, USA). To determine the level of nonspecific binding, fluorochrome conjugated isotype control antibodies were used. Flow cytometry was performed using a FACSCanto flow cytometer (BD Biosciences, San Jose, CA, USA).

\section{Osteogenic differentiation}

SHED was expanded in culture medium with FBS or xeno-free medium and seeded into commercial osteogenic differentiation medium (HyClone $^{\mathrm{TM}}$ AdvanceSTEM $^{\mathrm{TM}}$ Osteogenic Differentiation Kit, Hyclone) in 6-well plates. During differentiation, medium was completely changed every 3 to 4 days. After 28 days, calcium depositions were demonstrated in the extracellular matrix by staining with $40 \mathrm{mM}$ Alizarin Red S solution (Sigma), pH 4.1, for 20 min at RT, followed by rinsing three times with deionized water. The differentiated cells were observed using an inverted microscope and images were taken.

\section{Chondrogenic differentiation}

SHED was transferred into medium consisted of D-MEM, 10\% FBS, 10 ng/ml TGF- $\beta 1$ (sigma), 100 nmol/L dexamethasone (sigma), $6.25 \mu \mathrm{g} / \mathrm{ml}$ insulin (sigma), 110 $\mathrm{mg} / \mathrm{L}$ sodium pyruvate (sigma) and $1 \%$ penicillin/streptomycin in 24-well plates to induce chondrocyte differentiation. The medium was changed 3 times per week. After 21 days, proteoglycans were examined by staining with Alcian blue (Sigma), pH 2.5, for $20 \mathrm{~min}$ at RT, followed by three washes in tap water to remove unspecific binding of the dye. The differentiated cells were observed using an inverted microscope and images were taken.

\section{Spiral ganglion neurons differentiation}

SHED were seeded onto poly-L-ornithine (Sigma) and laminin (Sigma) coated 24-well plates. The cells were cultured for 14 days in spiral ganglion neurons induction medium consisting of DMEM/F12, $20 \mathrm{ng} / \mathrm{ml}$ neurotrophins brain-derived neurotrophic factor (BDNF), $20 \mathrm{ng} / \mathrm{ml} \mathrm{neu-}$ rotrophin-3 (NT-3), $20 \mathrm{ng} / \mathrm{ml}$ glial cell-derived neurotrophic factor (GDNF) (all from Sigma), supplemented with 2\% N-2 (Gibco), 2\% B-27 supplement, $100 \mathrm{U} / \mathrm{ml}$ Penicillin, and $100 \mu \mathrm{m} / \mathrm{ml}$ Streptomycin. The medium was changed every other day. The differentiated cells were stained with $\beta$-tubulin III (Tuj1; BioLegend, San Diego, CA, USA) and Tropomyosin receptor kinase B (TrkB; Santa Cruz Biotechnology, Inc, Dallas, TX, USA). Nuclei were counterstained with $20 \mu \mathrm{g} / \mathrm{ml}$ 4,6-diamidino-2-phenylindole (DAPI; Sigma).

\section{Statistical analysis}

All experiments in this study were performed at least 3 times. The data shown are representative results. Values were calculated as the means and standard deviations. Statistical tests were analyzed by one-way ANOVA of variance using SPSS software (Version 10.0; SPSS, Chicago, IL, USA). The values at $\mathrm{p}<0.05$ were deemed significant.

\section{Results}

\section{Effects of xeno-free culture conditions on the morphology and proliferation capacity of SHED}

To test the ability of xeno-free medium (MSC Growth Medium DXF, MSCGM-CD Bulletkit and Xuri MSC Medium Serum-free/Xeno-free) to support the growth of human SHED, first the morphology of three SHED lines (SHED \#1, \#2, and \#3) cultured in xeno-free medium was examined. SHED was extracted from the dental pulp of exfoliated deciduous teeth using outgrowth method. After $7 \sim 14$ days, spindle shaped cells were seen migrating
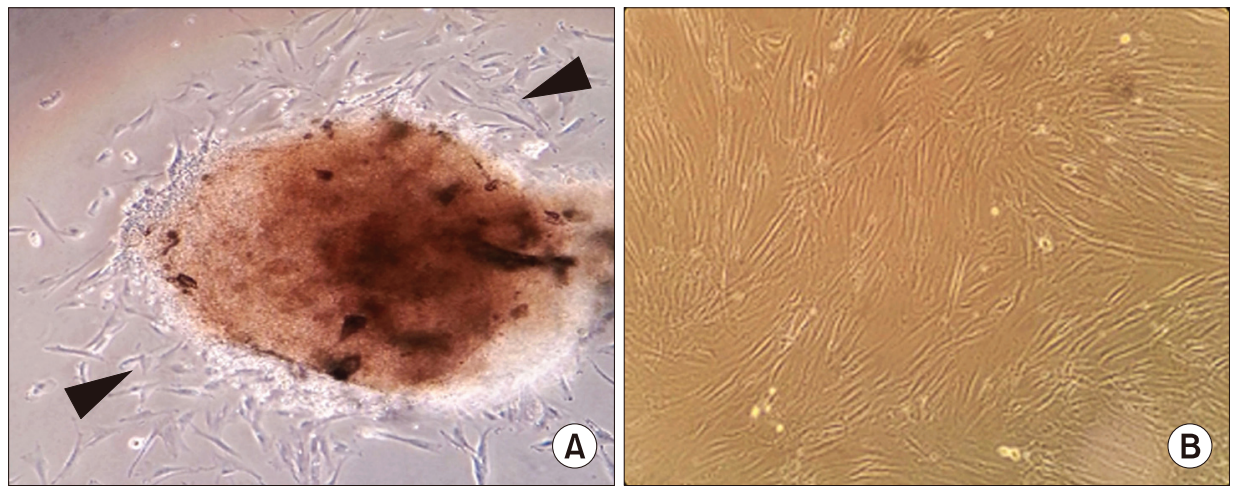

Fig. 1. Isolation and morphological observation of stem cells from pulp tissue of human deciduous teeth. (A) Outgrowth of stem cells (arrow head) from dental pulp tissue of human exfoliated deciduous teeth. (B) Phasecontrast images showing a fibroblast-like morphology of the in vitro growth SHEDs. Magnification: $40 \times$. SHED, stem cell from human exfoliated deciduous teeth. 
from pulp tissue into the flask (Fig. 1A) and reached 80 $90 \%$ confluence by 25 days in culture medium with $10 \%$ FBS (Fig. 1B). At passages 3 4, cells were adapted to the xeno-free conditions by gradually increasing the concentration of xeno-free medium and decreasing the concentration of the SHED culture medium with FBS every second day during the adaptation phase. The morphology of cells growing in MSCGM-CD medium was similar as those cultured under serum containing medium (Figs. 2A, B). The growth of all SHED lines showed elongated spindle shaped morphology. SHED morphological changes

A

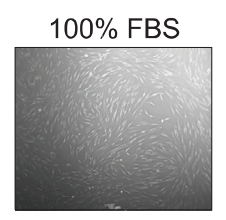

B

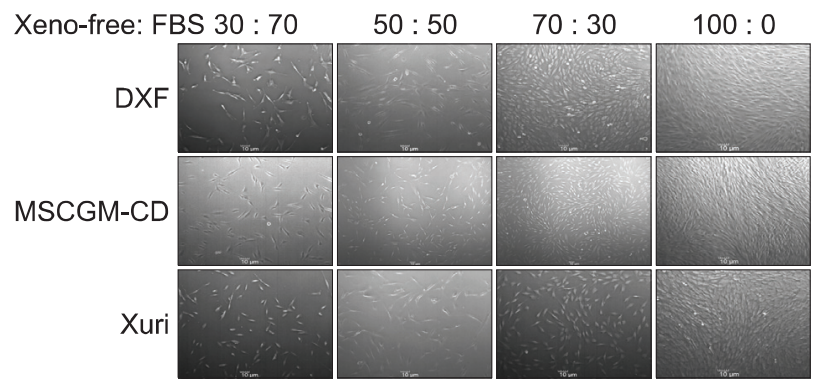

C

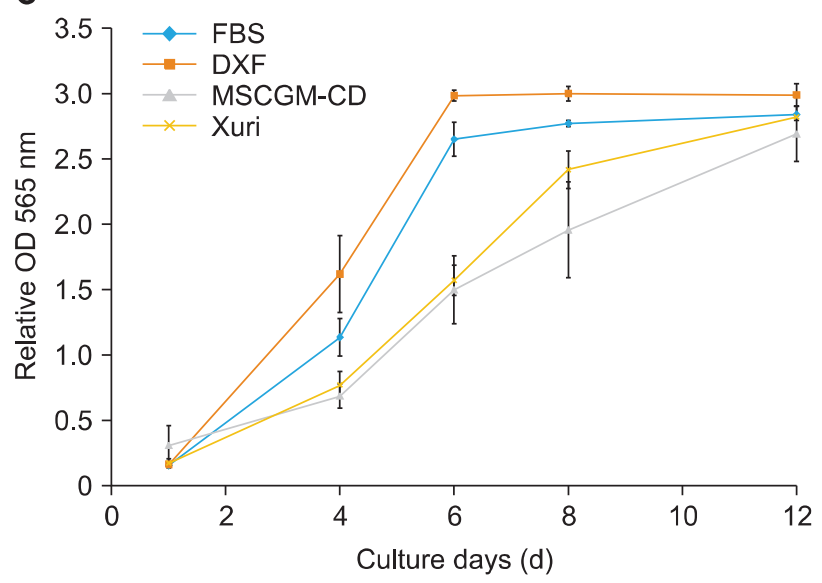

Fig. 2. Morphology of SHED during the adaptation to xeno-free medium. (A) SHED cultured with FBS-containing medium. (B) The representative photomicrographs showing the morphology of SHED cultured in xeno-free media. (C) Representative graphs of cell proliferation in four different conditions. Scale bar $=10 \mu \mathrm{m}$. SHED, stem cell from human exfoliated deciduous teeth, FBS, fetal bovine serum, DXF, MSC Growth Medium DXF, MSCGM-CD, MSCGM-CD Bulletkit, Xuri, Xuri MSC Medium Serum-free/Xeno-free. were observed to begin during the adaptation process with ratios of DXF or Xuri medium to culture medium with FBS at 70:30 (Fig. 2B). The cells expanded in DXF medium also displayed a fibroblast like morphology but had short elongated bipolar length, cells grown in Xuri exhibited polygonal shape with elongated bipolar or multipolar morphology (Fig. 2B).

To further determine the proliferation rate of SHED grown in xeno-free medium as compared to serum-containing medium, the cells were seeded with the same cell

A
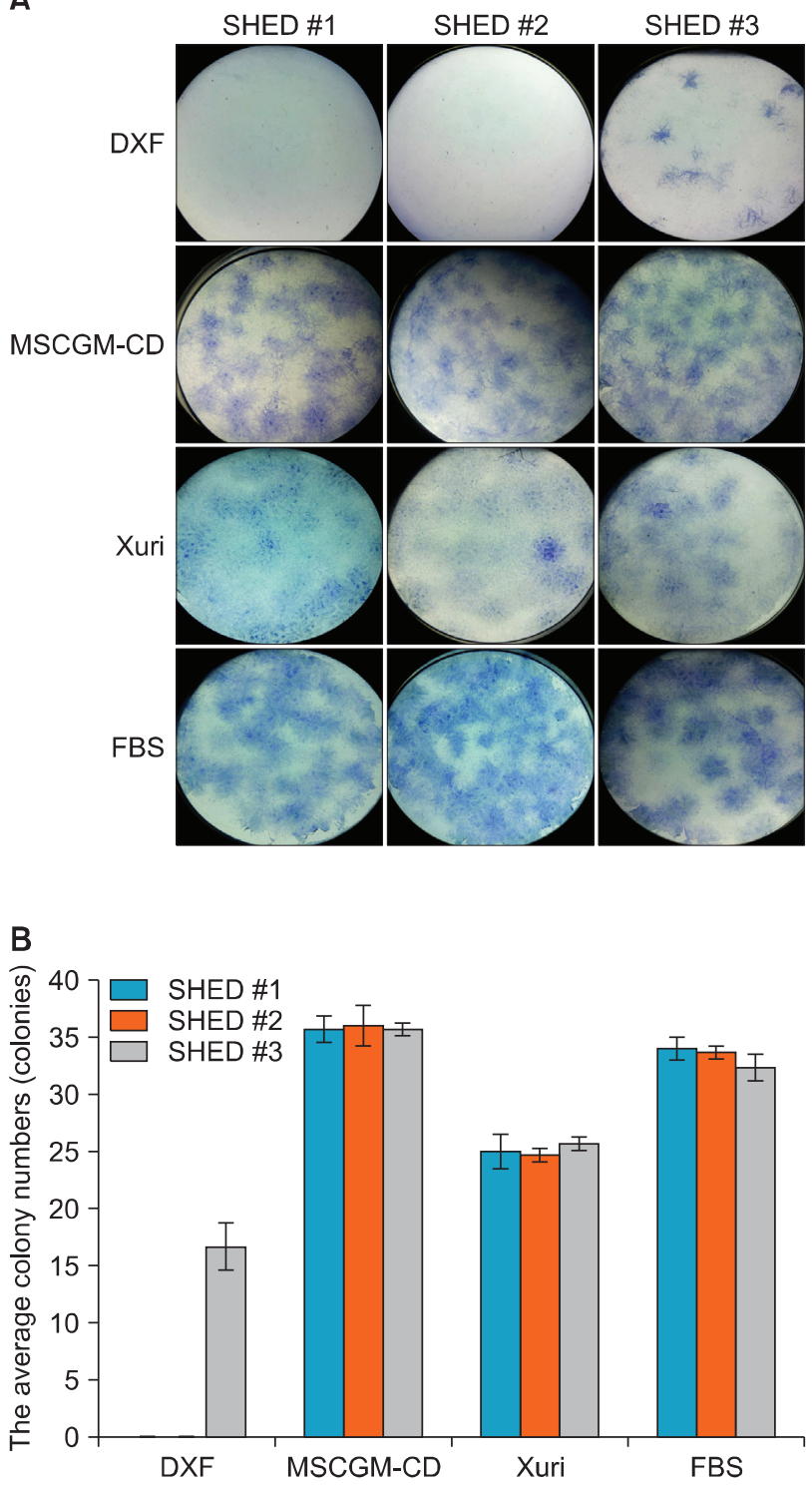

Fig. 3. Colony-forming unit-fibroblast assay of xeno-free expanded SHED. (A) Representative images of the colonies stained by toluidine blue after 14 days of cultured in four different conditions. (B) The total number of colonies counted within each condition. SHED, stem cell from human exfoliated deciduous teeth. 
seeding density and then collected at several time points (Day 1, 4, 6, 8, and 12). SHED in all media had similar trend to the growth curve (Fig. 2C). The proliferation analysis showed that at Day 4 the cultures with DXF medium clearly exhibited higher proliferation rates of SHED as compared to other media (Fig. 2C). Subsequently, SHED continued to proliferate at a highest rate in DXF medium at Day 6, 8, and 12. The analysis verified that serum-containing medium was superior to Xuri and MSCGM-CD system, respectively in supporting the growth of SHED (Fig. 2C).

\section{Effects of xeno-free culture conditions on colony- forming unit-fibroblast (CFU-F) assay of SHED}

Historically, CFU-F assay has been used as a correlative assay to determine the quality of cell preparations. To test the potential of SHED expanded in xeno-free medium, three SHED lines (SHED \#1, \#2, and \#3) were seeded at 500 cells/well of 6-wells plate and further cultured for 14 days (Fig. 3). All counted colonies were larger than $2 \mathrm{~mm}$ in diameter. All SHED lines expanded in DXF medium, but SHED \#1 and SHED \#2 did not play any important role in colony formation (Fig. 3A). The average number of SHED \#3 colonies (from 3 wells of 6-well plate) in DXF was $16 \pm 0.58$ (Fig. 3B). MSCGM-CD medium led to higher average colony numbers $(37 \pm 1.09)$ than that of other xeno-free medium and FBS-containing medium of all SHED lines (Fig. 3B). The average number of the SHED colonies in Xuri medium $(25 \pm 0.97)$ decreased as compared to the FBS-containing medium (33 \pm 1.12$)$ (Fig. 3B).
These data suggested that SHED exhibited maximum colony formation when cultured in MSCGM-CD medium.

\section{Effects of xeno-free culture conditions on the immune phenotype of SHED}

To further test the phenotype of SHED expanded in xeno-free medium, cell-surface antigens were analyzed by flow cytometry (Table 1). The results were consistent in all examined SHED lines and with repeated experiments. Three SHED lines were negative for the expression of the hematopoietic markers $\mathrm{CD} 34(<0.4 \%)$ in all media and positive for the expression of the MSCs markers CD44, CD73, and CD146 in MSCGM-CD, DXF, and FBS-containing media. In all SHED lines in xeno-free media, the expression level of CD44, CD73, and CD146 reduced as compared to FBS-containing medium (Table 1). The SHED expanded in MSCGM-CD medium displayed higher levels of the three MSCs markers as compared with SHED expanded in DXF medium. The immunophenotype CD44 (6.9 $\pm 0.50 \%)$ and CD73 $(8.9 \pm 0.60 \%)$ exhibited very low expression, and CD146 $(<1.8 \%)$ was absent in Xuri medium. Therefore, xeno-free medium used for subsequent experiment was MSCGM-CD medium.

\section{Effects of xeno-free culture conditions on the multipotent differentiation capacity of SHED}

Initially, to demonstrate the osteogenic differentiation of SHED expanded in MSCGM-CD xeno-free medium, the cells were induced in commercial osteogenic differentiation medium, which were analyzed with alizarin-red

Table 1. Surface marker expression characteristics of SHED in presence of different culture media

\begin{tabular}{|c|c|c|c|c|c|}
\hline \multirow{2}{*}{ Medium } & \multirow{2}{*}{ Markers } & \multicolumn{4}{|c|}{ Surface marker expression (\%) } \\
\hline & & SHED \#1 & SHED \#2 & SHED \#3 & Mean $\pm S D$ \\
\hline \multirow[t]{4}{*}{ FBS } & CD34 & 0.4 & 0.1 & 0.6 & $0.4 \pm 0.25$ \\
\hline & CD44 & 94.3 & 92.8 & 91.8 & $93.0 \pm 1.26$ \\
\hline & CD73 & 95.3 & 92.2 & 91.3 & $93.0 \pm 2.10$ \\
\hline & CD146 & 51.7 & 57.4 & 52.1 & $53.7 \pm 3.18$ \\
\hline \multirow[t]{4}{*}{ DXF } & CD34 & 0.2 & 0.1 & 0.1 & $0.13 \pm 0.06$ \\
\hline & CD44 & 30.9 & 28.6 & 30.3 & $29.9 \pm 1.19$ \\
\hline & CD73 & 61.6 & 62 & 63 & $62.2 \pm 0.72$ \\
\hline & CD146 & 33.7 & 30.1 & 30.2 & $31.3 \pm 2.05$ \\
\hline \multirow[t]{4}{*}{ MSCGM-CD } & CD34 & 0.3 & 0.2 & 0.3 & $0.27 \pm 0.06$ \\
\hline & CD44 & 84.3 & 85.4 & 87.1 & $85.6 \pm 1.41$ \\
\hline & CD73 & 84.7 & 82.0 & 81.5 & $82.7 \pm 1.72$ \\
\hline & CD146 & 51.4 & 52.6 & 51.3 & $51.8 \pm 0.72$ \\
\hline \multirow[t]{4}{*}{ Xuri } & CD34 & 0.2 & 0.3 & 0.2 & $0.2 \pm 0.06$ \\
\hline & CD44 & 6.9 & 6.4 & 7.4 & $6.9 \pm 0.50$ \\
\hline & CD73 & 9.5 & 8.3 & 9.0 & $8.9 \pm 0.60$ \\
\hline & CD146 & 0.9 & 1.8 & 1.5 & $1.4 \pm 0.46$ \\
\hline
\end{tabular}



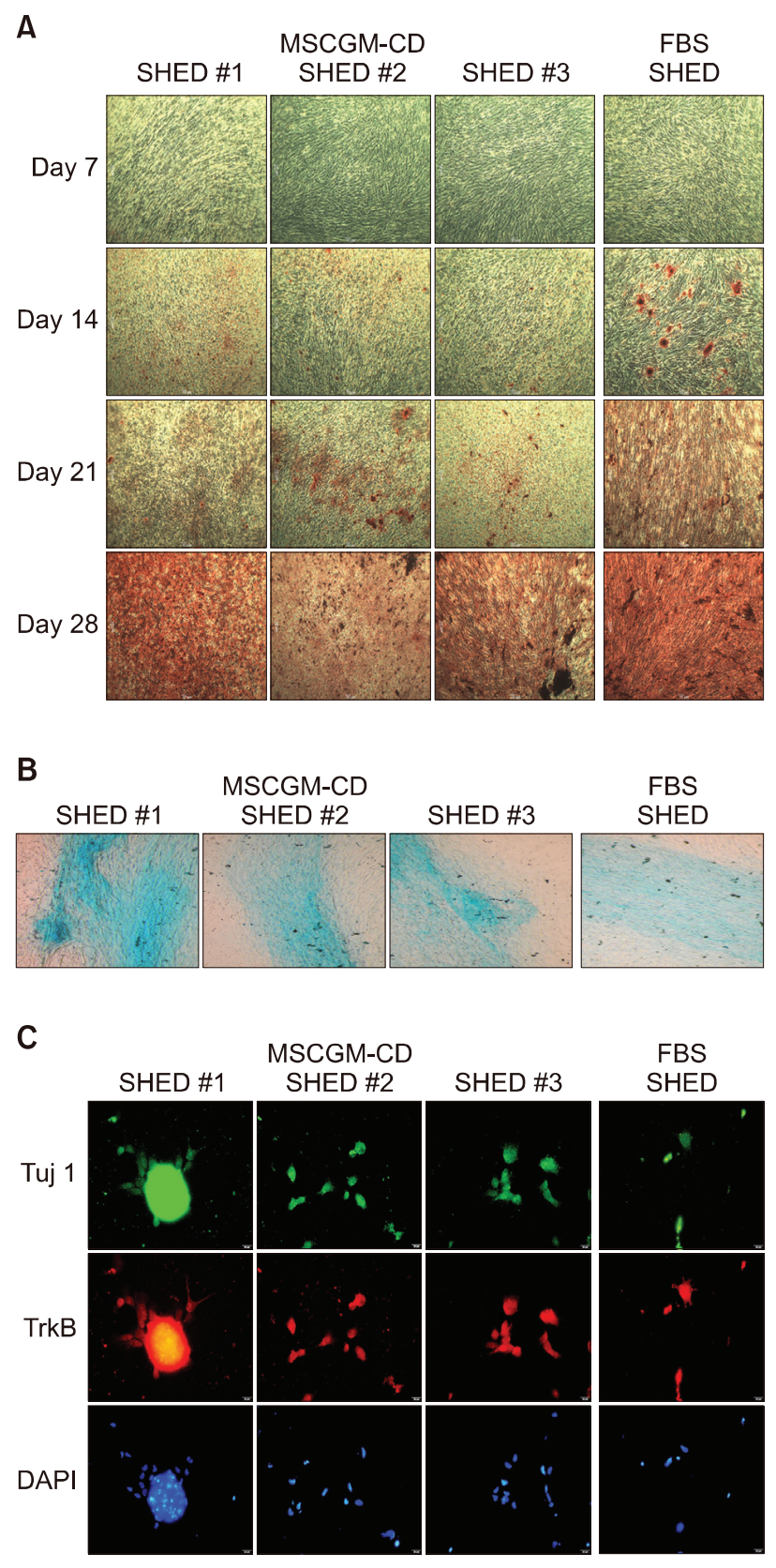

Fig. 4. Multipotent differentiation ability of SHED cultured in MSCGM-CD or FBS-containing medium. (A) Phase contrast images of SHED derived osteoblast-like cells, as shown by calcified nodules formation which appeared during alizarin red staining. (B) Alcian blue staining showed proteoglycans in chondrocyte cultures differentiated from SHED grown in both media. (C) After induction with spiral ganglion neurons induction medium for 14 days. The expression of the mature neuronal marker Tuj1 (green) and spiral ganglion neurons markers TrkB (red) is shown. Cell nuclei were counterstained with DAPI (blue). Magnification: A, 40×; B, 100×; C, Scale bar $=20 \mu \mathrm{m}$. SHED, stem cell from human exfoliated deciduous teeth, Tuj1, $\beta$-tubulin III, TrkB, tropomyosin receptor kinase B, DAPI, 4',6-diamidino-2-phenylindole. staining at Day 7, 14, 21, and 28. As shown in Fig. 4A, all 3 SHED lines grown in MSCGM-CD retained the ability to differentiate into osteoblast, as shown by calcified nodules formation which appeared during alizarin red staining. SHED changed the morphology from spindle shape, but there were no alizarin red stained cells on Day 7 of differentiation in both growing xeno-free and serum-containing media. At 14 days, similar observations were noted that cells started to form calcified nodules and were positively stained for alizarin-red on the MSCGM-CD and serum-containing media. However, the size and number of the nodules from MSCGM-CD was smaller than those from serum-free medium (Fig. 4A). Subsequently, from Day 21 until Day 28, there were increased numbers of positively stained nodules. At Day 21, the amount of alizarin red-stained cells of MSCGM-CD were still lower than the serum-containing medium. However, the staining was positive in $100 \%$ of alizarin red from SHED which expanded in both media on Day 28 (Fig. 4A).

To demonstrate chondrogenesis, the SHED were placed into chondrogenic medium for 21 days. All SHED lines expanded in both MSCGM-CD xeno-free and serum-containing media had the capacity to differentiate into chondrocytes (Fig. 4B). The differentiated cells changed their appearance from fibroblast-like into cobblestone-like cells. Moreover, the cells on the MSCGM-CD medium showed cartilage-specific proteoglycan and were positive for Alcian blue staining, similar observations on serum-containing media (Fig. 4B).

Fourteen days after transfer into spiral ganglion neurons induction medium, the morphology of SHED grown in both MSCGM-CD xeno-free and serum-containing media changed from elongated to extension of the cell body into neuron-like processes and a spherical soma (Fig. 4C). The SHED on both media types expressed mature neuronal marker Tuj1 and specific protein marker of spiral ganglion neurons TrkB. All cell nuclei were positive for DAPI (Fig. 4C).

The above data showed that SHED lines cultured in MSCGM-CD xeno-free medium still maintained their multilineage potential.

\section{Discussion}

Stem cells from the dental pulp of deciduous teeth has opened a new window for harvesting young stem cells and has offered a very promising therapeutic approach in regenerative medicine $(1,4,9)$. In order to achieve the production of safe and clinical grade SHED in sufficient quantities, all animal-derived materials that pose a poten- 
tial risk for infection transmitted by animal pathogens must be eliminated. Although FBS supplemented media have been added for fulfillment of growth factors, there is a risk of transmission of zoonotic diseases along with immunological allergic reactions (10). Hence, xeno-free system for isolation and expansion of cells has been introduced (11-13).

There are only a few optimal non-animal origin media for supporting MSCs from pulp of human exfoliated deciduous teeth growth (14-16). In this study, three xeno-free media, MSC Growth Medium DXF, MSCGM-CD Bulletkit, and Xuri MSC Medium Serum-free/Xeno-free were selected to investigate cell expansion potential, character properties, and multilineage differentiation of SHED in comparison with FBS containing media. SHED can be cultured robustly in MSCGM-CD Bulletkit system while maintaining their stem cell characters. In addition, SHED maintain their osteogenic, chondrogenic and neurogenic differention potential, which were comparable with SHED cultured in FBS-supplemented medium. Cells from pulp of human permanent teeth (hDPCs) culture under MSCGM$\mathrm{CD}$ condition can be reprogrammed to generate induced pluripotnet stem (iPS) cells which show similar pluripotent gene expression patterns comparing to those observe with FBS (17). The MSCs Growth Medium DXF and Xuri MSC Medium Serum-free/Xeno-free were also able to grow SHED but the morphology was changed due to adaptative process to the test media. There was variation in growth rates of cells cultured in 3 different xeno-free media. All three donor-derived SHED lines had the highest growth rate in DXF medium. Human adipose-derived stem cells were successfully grown in DXF medium (18). Human marrow MStroC has been cultured in Xuri medium with a redution in growth rate comparable to that in FBS-supplemented medium (19). It is suggested that each type of MSCs had similar requirments for nutrients, including growth factors and adhesion molecules.

SHED cultured under MSCGM-CD condition showed higher colony counts than other medium types, including serum-containing medium. However, hDPCs in MSCGM-CD exhibited significantly less primary colony formation than medium with FBS (17). Similar to MStroC cells (19), SHED colony forming ability in Xuri medium was lower than that observed with conventional FBS containing medium in all SHED lines. DXF medium could support the growth of all three donor-derived SHED lines but two of those lines failed to form CFU-F. One SHED line was less in CFU-F than that shown in FBS medium. For human bone marrow MSC propagation, it was reported that the plating MSC density could affect the proliferation and stemness preservation (20). It might be suggested that seeding densities is a fundamental parameter for SHED expansion. However, the exact reason for this difference in the growth pattern is unknown, but it could be that each donor or type of MSCs had its own optimum requirement for growth factors. Human bone marrow MSCs expanded in medium supplemented with recombinant human basic fibroblast growth factor (bFGF), platelet-derived growth factor-BB (PDGF-BB), and transforming growth factor (TGF)- $\beta 1$ showed extensive growth with retained phenotypic, differentiation, and CFU potential (21). PDGF-BB and bFGF have positive effects on proliferation in individual cells. However, TGF- $\beta 1$ appeared to provide no enhancement of cell growth on its own. The combination of any two growth factors could provide minimal or no significant enhancement of cell proliferation compared with single factors, and the combination of all three factors could provide a conspicuous synergistic effect (22).

A population of multipotent human MSCs must possess a specific cell-surface antigen expression profile (23). SHED in DXF or MSCGM-CD medium displayed positive expression of CD44, CD73, and CD146 and negative expression of CD34 (hematopoietic marker). Evaluation of different xeno-free culture media for all three donor-derived SHED lines revealed that those based on MSCGM-CD Bulletkit system was more suitable for expansion and little devoid of any alteration in immune phenotypes. CD44 is widely expressed in many types of human tissues, including osteoblasts and bone (24). Moreover, CD73 generated adenosine positively regulated osteoblast differentiation (25) and the deletion of CD73 in mice decreased osteoblast differentiation (26). Therefore, MSCGM-CD medium was able to maintain their multilineage differentiation capacity, especially into osteoblastic lineage of all three SHED lines.

\section{Conclusion}

The study concluded that SHED in MSCGM-CD Bulletkit culture system maintained the essential properties, such as spindle-shaped fibroblast morphology, surface MSCs markers, and multilineage differentiaiton potential. Future studies should focus on validation of these system to demonstrate long-term culture, multipotency, and genetic stability both in vtro and in vivo.

\section{Acknowledgments}

We would like to thank Dr. Nisarat Ruangsawasdi from Faculty of Dentistry, Mahidol University for her helpful suggestion and technical assistance. We also are grateful 
to Dr. Binito Shrestha for helpful discussions and critical reading of the manuscript. This work was supported by Mahidol University.

\section{Potential Conflict of Interest}

The authors have no conflicting financial interest.

\section{References}

1. Miura M, Gronthos S, Zhao M, Lu B, Fisher LW, Robey PG, Shi S. SHED: stem cells from human exfoliated deciduous teeth. Proc Natl Acad Sci U S A 2003;100:5807-5812

2. Demarco FF, Conde MC, Cavalcanti BN, Casagrande L, Sakai VT, Nör JE. Dental pulp tissue engineering. Braz Dent J 2011;22:3-13

3. Nör JE. Tooth regeneration in operative dentistry. Oper Dent 2006;31:633-642

4. Potdar PD, Jethmalani YD. Human dental pulp stem cells: Applications in future regenerative medicine. World J Stem Cells 2015;7:839-851

5. Mannello F, Tonti GA. Concise review: no breakthroughs for human mesenchymal and embryonic stem cell culture: conditioned medium, feeder layer, or feeder-free; medium with fetal calf serum, human serum, or enriched plasma; serum-free, serum replacement nonconditioned medium, or ad hoc formula? All that glitters is not gold! Stem Cells 2007;25:1603-1609

6. Ankrum J, Karp JM. Mesenchymal stem cell therapy: Two steps forward, one step back. Trends Mol Med 2010;16: 203-209

7. Halme DG, Kessler DA. FDA regulation of stem-cell-based therapies. N Engl J Med 2006;355:1730-1735

8. Spees JL, Gregory CA, Singh H, Tucker HA, Peister A, Lynch PJ, Hsu SC, Smith J, Prockop DJ. Internalized antigens must be removed to prepare hypoimmunogenic mesenchymal stem cells for cell and gene therapy. Mol Ther 2004;9:747-756

9. Kerkis I, Caplan AI. Stem cells in dental pulp of deciduous teeth. Tissue Eng Part B Rev 2012;18:129-138

10. Tonti GA, Mannello F. From bone marrow to therapeutic applications: different behaviour and genetic/epigenetic stability during mesenchymal stem cell expansion in autologous and foetal bovine sera? Int J Dev Biol 2008;52:10231032

11. Stute N, Holtz K, Bubenheim M, Lange C, Blake F, Zander AR. Autologous serum for isolation and expansion of human mesenchymal stem cells for clinical use. Exp Hematol 2004;32:1212-1225

12. Zhang D, Mai Q, Li T, Huang J, Ding C, Jia M, Zhou C, $\mathrm{Xu} \mathrm{Y}$. Comparison of a xeno-free and serum-free culture system for human embryonic stem cells with conventional culture systems. Stem Cell Res Ther 2016;7:101

13. Miyagi-Shiohira C, Kobayashi N, Saitoh I, Watanabe M, Noguchi Y, Matsushita M, Noguchi H. Evaluation of serum-free, xeno-free cryopreservation solutions for human adipose-derived mesenchymal stem cells. Cell Med 2016; 9:15-20

14. Bieback K, Hecker A, Kocaömer A, Lannert H, Schallmoser K, Strunk D, Klüter H. Human alternatives to fetal bovine serum for the expansion of mesenchymal stromal cells from bone marrow. Stem Cells 2009;27:2331-2341

15. Ben Azouna N, Jenhani F, Regaya Z, Berraeis L, Ben Othman T, Ducrocq E, Domenech J. Phenotypical and functional characteristics of mesenchymal stem cells from bone marrow: comparison of culture using different media supplemented with human platelet lysate or fetal bovine serum. Stem Cell Res Ther 2012;3:6

16. Bieback K, Kern S, Kocaömer A, Ferlik K, Bugert P. Comparing mesenchymal stromal cells from different human tissues: bone marrow, adipose tissue and umbilical cord blood. Biomed Mater Eng 2008;18(1 Suppl):S71-S76

17. Takeda-Kawaguchi T, Sugiyama K, Chikusa S, Iida K, Aoki H, Tamaoki N, Hatakeyama D, Kunisada T, Shibata T, Fusaki N, Tezuka K. Derivation of iPSCs after culture of human dental pulp cells under defined conditions. PLoS One 2014;9:e115392

18. Pelikant-Malecka I, Kaniewska-Bednarczuk E, Szrok S, Sielicka A, Sledzinski M, Orlewska C, Smolenski RT, Slominska EM. Metabolic pathway of 4-pyridone-3-carboxamide- $1 \beta$-d-ribonucleoside and its effects on cellular energetics. Int J Biochem Cell Biol 2017;88:31-43

19. Gerby S, Attebi E, Vlaski M, Ivanovic Z. A new clinical-scale serum-free xeno-free medium efficient in ex vivo amplification of mesenchymal stromal cells does not support mesenchymal stem cells. Transfusion 2017;57:433-439

20. Colter DC, Sekiya I, Prockop DJ. Identification of a subpopulation of rapidly self-renewing and multipotential adult stem cells in colonies of human marrow stromal cells. Proc Natl Acad Sci U S A 2001;98:7841-7845

21. Ng F, Boucher S, Koh S, Sastry KS, Chase L, Lakshmipathy U, Choong C, Yang Z, Vemuri MC, Rao MS, Tanavde V. PDGF, TGF-beta, and FGF signaling is important for differentiation and growth of mesenchymal stem cells (MSCs): transcriptional profiling can identify markers and signaling pathways important in differentiation of MSCs into adipogenic, chondrogenic, and osteogenic lineages. Blood 2008;112:295-307

22. Chase LG, Lakshmipathy U, Solchaga LA, Rao MS, Vemuri MC. A novel serum-free medium for the expansion of human mesenchymal stem cells. Stem Cell Res Ther 2010;1:8

23. Dominici M, Le Blanc K, Mueller I, Slaper-Cortenbach I, Marini F, Krause D, Deans R, Keating A, Prockop Dj, Horwitz E. Minimal criteria for defining multipotent mesenchymal stromal cells. The International Society for Cellular Therapy position statement. Cytotherapy 2006;8: 315-317

24. Hughes DE, Salter DM, Simpson R. CD44 expression in human bone: a novel marker of osteocytic differentiation. J Bone Miner Res 1994;9:39-44

25. Takedachi $M$, Oohara H, Smith BJ, Iyama M, Kobashi M, 
Maeda K, Long CL, Humphrey MB, Stoecker BJ, Toyosawa S, Thompson LF, Murakami S. CD73-generated adenosine promotes osteoblast differentiation. J Cell Physiol 2012;227: $2622-2631$
26. Cavaliere F, Donno C, D'Ambrosi N. Purinergic signaling: a common pathway for neural and mesenchymal stem cell maintenance and differentiation. Front Cell Neurosci 2015; 9:211 